\title{
Structural design considerations for an 8-m space telescope
}

\author{
William R. Arnold Sr. ${ }^{a}$, H. Philip Stahl ${ }^{\text {b }}$ \\ ${ }^{a}$ Jacobs ESTS Group, Marshall Space Flight Center, Huntsville, AL USA 35812; \\ ${ }^{b}$ NASA, Marshall Space Flight Center, Huntsville, AL USA 35812
}

\begin{abstract}
NASA's upcoming ARES V launch vehicle, with its' immerse payload capacities (both volume and mass) has opened the possibilities for a whole new paradigm of space observatories. It becomes practical to consider a monolith mirror of sufficient size to permit significant scientific advantages, both in collection area and smoothness or figure at a reasonable price. The technologies and engineering to manufacture and test 8 meter class monoliths is mature, with nearly a dozen of such mirrors already in operation around the world. This paper will discuss the design requirements to adapt an $8 \mathrm{~m}$ meniscus mirror into a Space Telescope System, both launch and operational considerations are included. With objects this massive and structurally sensitive, the mirror design must include all stages of the process. Based upon the experiences of the Hubble Space Telescope, testing and verification at both component and integrated system levels are considered vital to mission success. To this end, two different component level test methods for gravity sag (the so call zero- gravity simulation or test mount) are proposed, with one of these methods suitable for the full up system level testing as well.
\end{abstract}

Keywords: ARES V, 8m Space Telescope, structural design, key considerations

\section{INTRODUCTION}

Traditional metrics for estimating the cost and complexity of a space telescope system need to be reconsidered due to the available volume and payload mass that an ARES V launch vehicle makes possible. The designer no longer needs to be an expert in Origami to fit the telescope and satellite into the shroud. The complexity and cost of ultra-light weight segmented-mirrors and their related structural components can now be put to better performance and reliability features.

This paper discusses the design problems associated with launching an 8 meter class thin meniscus mirror into space. First we will look into why one would want to do this then we will discuss what is necessary to accomplish the task.

\subsection{What is so good about a thin meniscus primary?}

Everyone knows that a space optic has to be light enough to be launched. In the past, a lot of performance compromises had to be made in the name of saving mass. For monolithic or single element primaries, that meant pocket milling the mirror blank or fabricating "eggcrate" mirrors either by fusion bonding or frit bonding techniques, often combined with uses of even more exotic materials as beryllium or silicon carbides instead of the better known ULE (glass) and ZERODUR (ceramic) low thermal expansion substrate materials. Thermal expansion during fabrication, test, and operation has been a major concern. The very process of light-weighting and the optical figuring of pocketed mirrors induce quilting effects which compromise the mid and high frequency wave front. The polishing process applies a nearly uniform pressure under the lap but the light-weighting pockets present a large variation in stiffness whenever a portion of that lap goes over a rib. The thin plate spanning the "cells" deflects under this pressure, so the glass is removed more over the rib than in the center of the cell. Once the lap pressure is removed, the center springs up causing the quilting effect. Smaller laps and spot polishing to lessen this effect in turn causes increased surface roughness. Larger laps are needed to smooth out this roughness, putting the quilting back into the part.

To achieve larger apertures, the primary can be broken into smaller units or segments as in the case of the James Web Space Telescope (JWST), which combines exotic materials and pocket milled light weighting with segmented mirrors to produce a 6.5 meter aperture mirror. The segment edge effects (diffraction and degraded point spread functions) due to the small gap which must exist between the individual elements and the fact that the figure cannot be controlled near the edge combine with the thermal expansion characteristics of the material to again compromise the wave front achievable, 
regardless of the quality of each individual segment. The challenges of radius matching, edge effects and segment orientation to high accuracy place severe limits on the achievable system wave front. Figure control during manufacture and the quilting due to pocket-web stiffness variations inevitably result in rougher mirrors. University of Arizona (Steward Observatory Mirror Lab) has pioneered smart laps, vacuum laps and numerous really clever innovations to try and overcome the inherent problems with eggcrates, without that much success to date. The complexity of packaging these segments into limited shroud volume, then reliably deploying and aligning everything back into the telescope operating configuration is just one of the many compromises that traditional space optical systems have had to deal with.

The quality of figure obtainable with a thin meniscus mirror is only limited by the spacing of the polishing and test mounts during fabrication. Using the same advanced "smart" lap and flexible lap technologies developed to overcome the quilting problems of pocketed mirrors, achieving the potential smoothness suitable for UV wave lengths has become practical. The coating technologies for 8 meter class optics will have to be refined to get suitable uniformity, but the basic understanding of how to handle the coating of an 8 meter mirrors is well understood.

\subsection{Mechanical advantages of thin meniscus mirrors}

Beyond the optical manufacturing advantages of a meniscus mirror, there are significant mechanical advantages as well. Eggcrate and other lightweight optics have large stiffness discontinuities and stress concentrations which limit their ability to withstand launch environments. The meniscus mirror has a uniform stiffness and smooth global bending characteristics therefore the main variable in launch stresses becomes the number of attachment points.

The true tensile strength of glass is on the order 3,000,000 psi. The traditional design limit of 1000-7000 psi is based upon subsurface damage (i.e. microscopic cracks left over from manufacture -- shaping and figuring) plus any internal flaws, inclusions and undetected handling damage. Up to a certain size (called obviously enough the "critical flaw size" for a given tensile/shear stress, the crack will not grow rapidly. Above that stress level the crack grows catastrophically. The whole point of successive grind and polish is to remove the layers of damage caused by one grit size while causing smaller and smaller fractures until you get well below the "critical" size. All glass/ceramics mirrors have these cracks; it is just a matter of how long until they grow to failure levels. The statistical nature of the random flaw distribution is why such low allowables are used. As we will be bonding to the outer surface, and the stresses induced in the glass from adhesive cure shrinkage, thermal expansion mismatch and applied loads will all be imparted on the glass in the first few millimeters of depth, this subsurface damage layer is the critical design limiter. Bigger pads mean lower applied load stress, but higher shrinkage and thermal stresses; hence it is as much an art as a science to design bond pads. The extremely thin bond lines add to the mix, making even analyzing these parts a true art.

Eggcrate and cast mirrors have additional problems with bond pad stresses. The issue is where to put them. The Kepler primary mirror had specially reinforced areas designed integrally with the support pads to distribute the loads into the mirror with minimal bending. Kodak (now ITT) likes to design pads for shear joints into the webs of eggcrates. Bonds in primarily shear are stronger than tensile types, but require pocketing the back face sheets (i.e. more expensive and time consuming). The cast mirrors have higher CTE non-uniformity as well as high average value and have the same stiffness issues, you need to design in attachment, or as UA SOML likes to have many, many attachment points.

\section{BASIC ELEMENTS OF A SPACE TELESCOPE}

Several things distinguish a space based observatory from its terrestrial counterpart. The most obvious are that it must survive launch and then operates completely by remote control (or even autonomous operations for extended periods of time). Unlike the Hubble Space Telescope, which was serviceable on orbit, the following generations of observatories are planned for L2 orbits (sun-earth-moon Lagrangian Points) where manned serving is not practical. The baseline planning for the ATLAST Observatory is for remote (i.e. robotic) serving missions. This consideration drives many design details. Figure 1 shows the what these elements look like for the ATLAST system. 


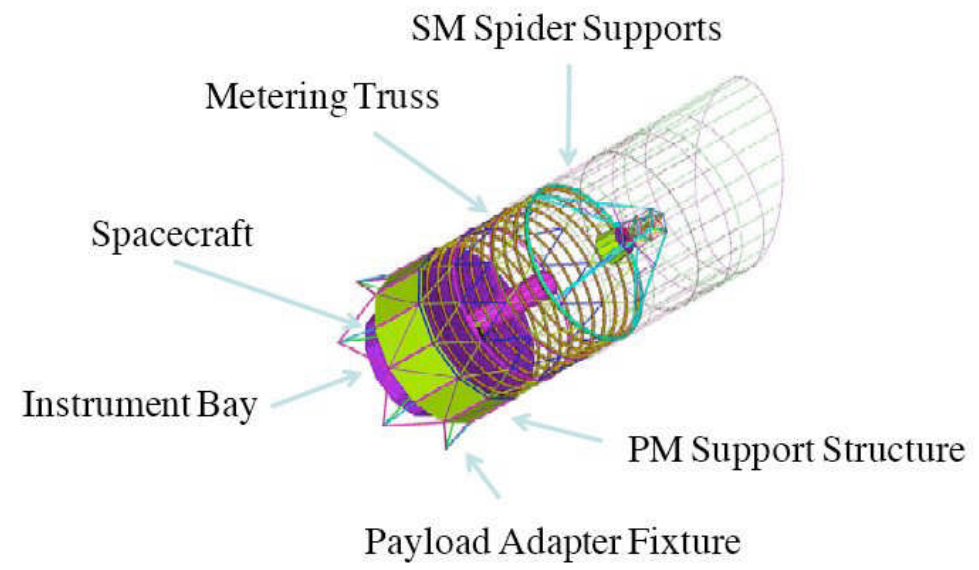

Fig. 1. This is the current structural design for ATLAST 8m Space Telescope (On axis version, dual field with 3 Foci). The system is divided into three functional areas; Optical Assembly, Spacecraft and Payload Adaptor.

\subsection{Optical Assembly (OA) Elements}

The optical assembly consists of the primary mirror (PM) and its operational support system, the main support (or base) truss, the forward (or metering) truss, the secondary spider truss, the secondary mirror assembly and the fixed aft optical assembly. In addition, the thermal control/protection and sun shield systems, aperture closure doors (in case of accidental near sun pointing situations) and internal stray light baffles are considered part of the OA. Figure 2 shows the basic optical layout.

A key element of the Optical assembly which only functions during launch, then must by design completely decouple from the primary for the remainder of the mission is the PM launch support system. This system becomes the major design driver for the structural design of the OA.

The fixed Aft Optical Assembly (AOA) splits and folds the optical path to the various planned and future scientific instruments as well as the fine guidance system sensors. While the baseline does not envision any active figure control of the primary mirror, and limited secondary mirror positional adjustments, the AOA has several locations were some form of flexible or active mirror could be inserted. These locations would lend themselves to robotic exchange of just that subassembly, should such a complex system fail during the extended mission.

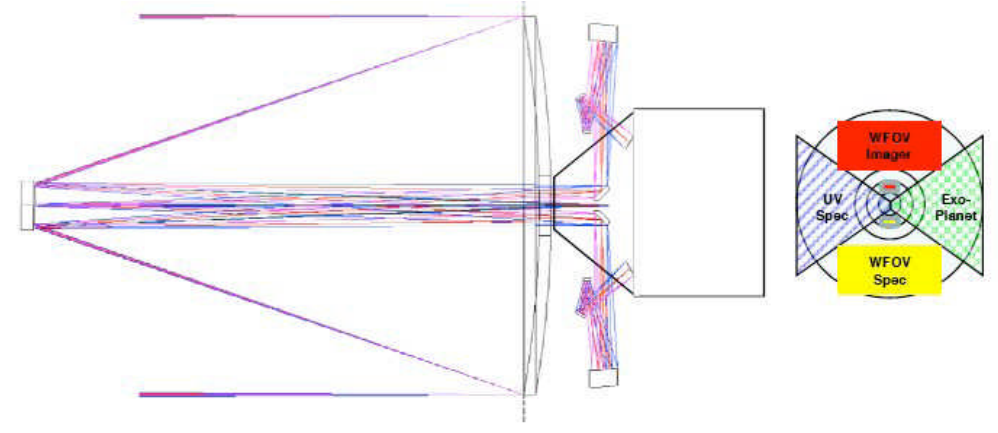

Fig. 2. This is the current optical design for ATLAST $8 \mathrm{~m}$ Space Telescope (On axis version, dual field with 3 Foci). The design studies for the system provide for a very flexible science mission, from planet finding to UV spectroscopy. 


\subsection{Satellite Elements}

The satellite portion of the spacecraft consists of the basic housekeeping functions, such as providing power, pointing and guidance, command and control, system monitoring and communications with earth. The scientific instruments attach to a garage interface on the satellite, which provides services to each instrument. The each instrument will have the capability to align itself with the fixed optical elements. Key sections of the main satellite are also "line replaceable", while a backbone of basic services will remain available during any robotic servicing mission. The satellite also has the final stage propulsion to insert into orbit or refine the orbit, the so called station keeping and momentum dump thrusters.

A departure from traditional spacecraft design is how the Optical Assembly and Satellite sections interface to the launch vehicle. In most previous designs, the Optical assembly would be mounted on the satellite bus and the bus structure would carry the launch induced loads through to the Interface, Adaptor or Attachment Ring of the vehicle. In the situation where the Optical assembly is 3-4 times as massive as the satellite and has a massive displacement driven truss spanning the complete diameter of the payload volume, it makes more sense to transmit the satellite load through that Base Truss. It does require local increases in the size of a number of truss members, but the total mass gain is far less than the structure required if all the inertial loading of the 26 metric ton primary and the other 15 or more tons of metering structure, baffling and shields had to be transmitted through the satellite's structure.

\subsection{Payload Adaptor Fixture}

This is the structural element(s) which attach the Observatory to the launch vehicle. With the ARES V and the large telescope envisioned this will most likely be an additional truss like structure, tying into the Base Truss geometry and distributing the load into the cylindrical portion of the upper stage. This structure is traditionally detached or jettisoned prior to final orbit insertion. Current studies are looking into the best staging approach to this, keep or discard. The current design baseline keeps the PAF and uses it to support components as shown in figure 3.

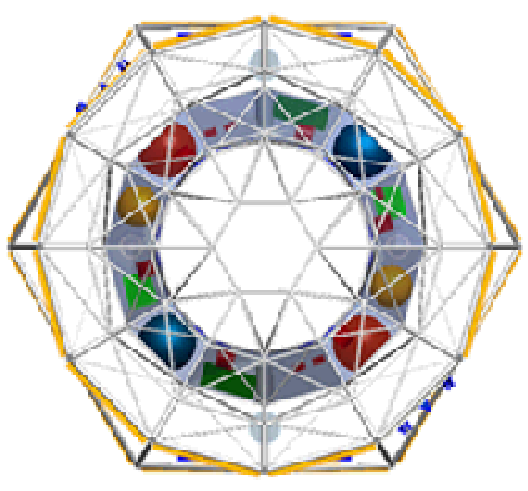

Titil vition

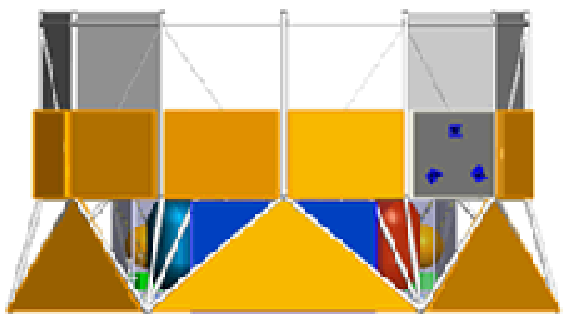

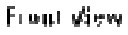

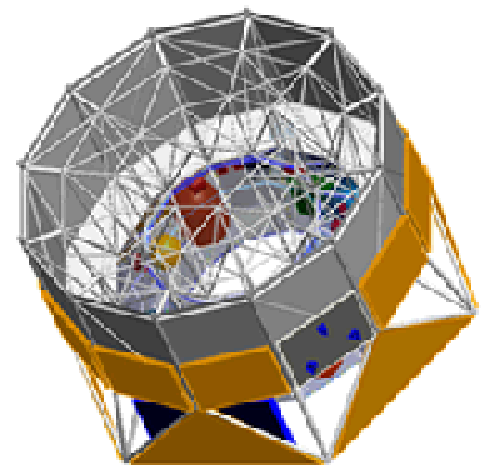

Is thien

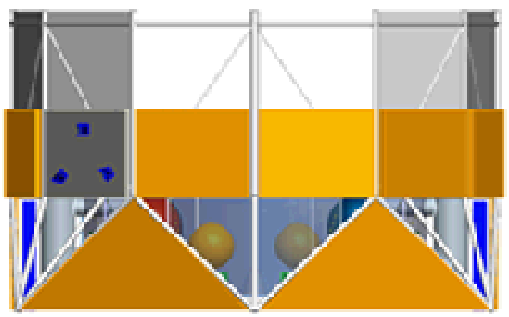

glile virow

Fig. 3. This is the current Modular Spacecraft design for ATLAST 8m Space Telescope. The solar panels are shown stored inside PAF and radiator panels and autonomous rendezvous and docking interfaces mounted outside of PAF structure. 


\section{DESIGN DRIVERS AND IMPORTANT ISSUES}

\subsection{Basic design drivers}

All components and elements of the observatory must satisfy these general requirements. The design process must find a suitable configuration which meets all of these conditions. There will always be volume and weight constraints no matter how large the booster is. There will always be just one more instrument or performance requirement which is beyond the current baseline. This paper reflects the lessons learned and decisions made over two design trade studies for an $8 \mathrm{~m}$ class telescope mission for the ARES $\mathrm{V}$ vehicle. These studies have resulted in the development of a number of tools and experiences unique to addressing issues relating to really large telescopes.

- Survive the launch environment

- Not interfere with Launch Vehicle control systems (Minimum frequency requirements)

- Be within the lift capacity of the vehicle and fit within the shroud dynamic envelop

- Get into operational configuration (deployment, release mechanisms, etc.)

- Function in operational environment (thermal, jitter and optical performance)

- Serviceable and/or sustainable over planned life (redundancy, replacement and replenishment)

\subsection{Primary mirror support systems have three main requirements;}

The most critical single component in the system, from both a cost and fragility view point is the primary mirror. In all previous space telescopes, the main optics has been the central driver in the design.

1. The support system must retain the mirror throughout the trajectory, while subject to accelerations, vibrations and acoustical loads. (survive launch) The allowable stress in the mirror is a function of surface finish on back and edges of the mirror. (see section 4.2)

2. The support system must couple or decouple the primary mirror as a mass-spring system from the frequency range of the launch vehicle major structural and control frequencies. Usually stated as 20 hertz for axial and 78 hertz lateral. Less defined is a torsional mode of the primary vibrating around the optical axis. While excitation levels for this mode are not defined, this mode can only be controlled by tangent bars or special features. (not interfere with launch vehicle dynamics or controls)

3. The support system must release the mirror to a kinematic state after achieving orbit; this kinematic system must control the position of the mirror during insertion and operational maneuvering. (get into and maintain operational configuration) If any of the launch system stays in contact with the primary, it will degrade the optical performance by causing bending in the mirror.

And the primary mirror and its support system(s) have to be designed as a unit. This is particularly true due to the size and mass of an $8 \mathrm{~m}$ monolithic mirror. Every move, every operation and every test requires massive equipment, space and control. The nature of glass is that it is unforgiving -- impact damage, improper support at any stage or numerous other conditions can result is total loss. And the high initial cost and lead time precludes back up mirror(s).

\subsection{Telescope Structure}

The base truss must support the primary mirror and satellite during launch then maintain optical alignment between the primary mirror and aft fixed optical assembly. The forward metering truss and SM spiders must support the secondary mirror, aperture doors, sun shields (in stowed position), and internal baffles during launch. The forward metering truss and SM spiders are the primary structure responsible for maintaining the alignment and spacing of the primary to secondary mirrors. The sun shields must deploy after launch and lock in place. The doors must be capable of opening and closing after launch, but the launch stow locks are only necessary during ascent. 


\subsection{Satellite Bus, Instrument Bays and Instrument Packages}

The satellite has two sections, the main (utilities supplier section) and the instrumentation bay section. The main section is initially locked to the base truss for launch. Once launch environment is over, the hard locks are released and the main section is isolated from the telescope structure. This is to minimize jitter due to reaction wheel unbalance, solar panel motion and antenna motion. The instrument bay section remains hard mounted to the telescope scope structure. The main design drivers of the instrument bays are maintaining optical path stability and ease of exchange of package robotically. The instrumentation bay must satisfy optical alignment as well as structural criteria. The "service" section of the satellite must be isolated from the optical portion to control jitter, this places both frequency and stability criteria on these items beyond the normal satellite duties.

\subsection{Testing and verification (How to accomplish with such massive components)}

While not always considered at the design stage, how the testing will be accomplished has to be designed into the hardware from the beginning. The size and complexity of any operation related to the observatory or its elements requires massive tooling and facilities. It becomes a major design driver, which you have to keep in mind at every decision and trade point.

There are a lot of issues related to testing which will take years to establish a consensus on how to best accomplish. The infrastructure and tooling will have to be developed and/or modified to deal with an optical system this large. While shaker tables exist which can handle 60-70 metric ton assemblies, it is not sure how one would protect the optical surface if the flight optics were to be used. If aluminum (has similar stiffness and mass characteristics as glass where substituted for the qualification of the structural elements (launch support and truss) deflections and strength, what testing of the actual flight optics (bond pad proof testing, low level vibration tests on base only, etc.) would be necessary to qualify the system for launch. At the time the system is being designed, the vehicle itself is being created, hence the "design loads" are prediction or best guesses. What should be the contingency plan if the environments become worse or far better when the actual vehicle data becomes available while the key components of the optics are being fabricated and tested. No one has data on how an $8 \mathrm{~m}$ mirror responses to the acoustic environment of an ARES V launch. One of the issues which need to be addressed is exactly how to scale existing methods to these large dimensions and volumes.

\subsection{Major Issues unresolved at this point in the design process}

Here is just a short list of major issues to be resolved are:

- Quasi-static and vibration loads (what are reasonable design loads for a vehicle not yet designed and how does one test these loads on such massive optical assemblies)

- Acoustic environment (once reasonable levels determined.) How will a large thin meniscus mirror respond acoustically in the ARES V shroud? This is beyond current database or experience. How would one test this?

- Mechanisms and replacement/servicing provisions (have to get it right)

\section{PRIMARY MIRROR AND SUPPORT SYSTEMS}

The design of the primary mirror launch support drives the complete design of the telescope assembly, and through the resulting truss geometry the rest of the satellite and instrument bays. The following sections will discuss the design trades and interaction of this variable (the number of axial support points for launch) with the whole telescope structural design.

\subsection{Desirable versus practical supports for space application}

All the existing (terrestrial) 8 meter primary mirrors are supported by two systems, a radial and an axial support system. These systems are hydraulic, designed to offset the weight of the mirror at various elevation angles against the fixed pull of gravity. These systems apply forces and rely on six hard points to define position. Glass mirrors do not respond well to high local loads, so these hard points usually have load limiting features (a breakaway feature - not usually a popular term with opticians). These axial systems in general can only deal with small tensile forces due to wind suction or seismic events. One of the biggest limitations of any mirror support is that you can only attach it on the perimeter or 
back face. Hydraulic support systems have the nice characteristic that they can be designed with limited sensitivity to the deflections of the support structure. Unfortunately, they also have another characteristic which eliminates them from consideration as a launch support system - they always leak. And the consequences of a major or even minor leak during ascent would compromise the whole observatory. The other traditional method of creating a kinematic axial support is the whiffle tree method. With the accelerations levels expected, the weight and complexity of such a system would be prohibitive. The initial trade studies did establish how many levels would be required, but no suitable support structure concept which would not weigh more than the 26 metric ton mirror itself was found. And while the ARES V has a large lift capacity, most of it would be used just for the mirror and its support.

The less desirable, but more practical approach was to have a very stiff support truss with softer axial spring elements. During launch, the accelerations and random vibrations cause the truss and mirror to flex as a unit. The mirror bends and flexes both on its own and together with the deformations of the truss (due to the stiffness of the launch support system connecting the two). On the plus side the depth of the truss and truss member orientation have more to do with controlling displacement than the mass alone. This is the reason so many bridges and large buildings are designed as trusses.

The key to a successive design is controlling the smoothness of the deflections in the primary mirror. The design trade becomes how many points and their distribution on the primary mirror and how uniformly these points are supported by the truss. The absolute deflection of the truss is coupled to the primary mirror by these springs, so minimizing the global bending induced in the mirror by deflections of the truss becomes one of the merit functions. While this would normally tend to drive the design to very soft spring rates, the additional constraint driven by vehicle dynamics is that the mass of the primary does not interfere with the launch vehicle control system which pushes the design towards stiffer spring rates.

\subsection{Relationship between backing truss and mirror attachment points}

The peak tensile stress in the glass is what determines the survival or failure of the mirror. This peak stress has several contributing sources. Global bending in the mirror causes tensile stresses at the top or bottom surfaces. The critical tensile stresses at the surface and within a few millimeters of the surface are due to a combination of global bending, local pad direct loads and bond/pad induced loads. This peak tensile stress can occur in one of two places, either at or near a radial bond pad or at or near an axial bond pad. The radial or edge support system is actually a bumper system which mainly applies compressive loads, the tangential vibration modes are not likely to be excited during launch, but these loads would interact with the radial pads as shear and bending loads. The bending portion of that radial loading would result in a tensile component of stress at the pad location. The more likely location is the axial pads (on the rear face of the mirror) which must resist both tension and compressive loads. While the axial quasi-steady state thrust of the launch vehicle causes mostly compressive loads, the random vibration can result in large tension loads on these pads.
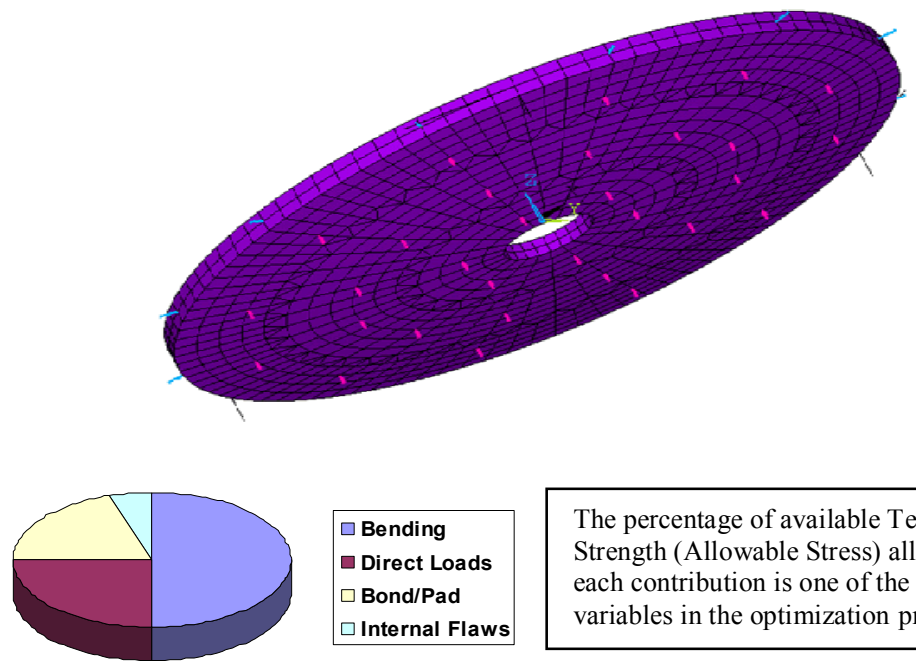

$\square$ Bending

$\square$ Direct Loads

$\square$ Bond/Pad

$\square$ Internal Flaws
The percentage of available Tensile Strength (Allowable Stress) allocated to each contribution is one of the design variables in the optimization problem. 
Fig. 4. The number and position of the axial and radial support points on the primary mirror for the ATLAST 8m Space Telescope are related to the truss they attach to. The main variable is the number of circumferential "cells" in the pattern. The radii of the patterns and the spacing behind the mirror must match to the truss geometry for maximum stiffness. The individual spring rates (at each radius) can be adjusted to balance the deflections of the truss within limits.

The design problem then becomes, find the number of points and a distribution of those points to minimize global bending stress and local pad induced stresses. Fewer pads means higher point loads and therefore larger pads which in turn means higher pad bond stress effects, i.e. less available stress capability to be allocated to global bending (hence a stiffer truss is required). More support points mean a more complex truss or stiffness loss to secondary structure to match point locations with joints in truss (high stiffness attachment locations). The response of the truss to mirror inertia loading and the bending stresses induced in the mirror combined with the local point load stress is another merit function. The pad size versus shrinkage stresses and thermal expansion stresses becomes a local minima search, once a global minimum is found. The total mass of the truss plus the sum of the unit masses of each point (pad, spring element, release mechanism and attachment structure) is the final merit value being minimized. As mentioned in an earlier section, the design allowable strength of the substrate is a function of surface preparation and any nearby internal flaws.

With a meniscus mirror (regardless of size), the whole back face can be processed with modern techniques to establish a very reasonable stress capability. Once polished out, the internal flaws (those significant in size to be important) near the back surface can be mapped and the pad location and orientation adjusted to avoid problem areas.
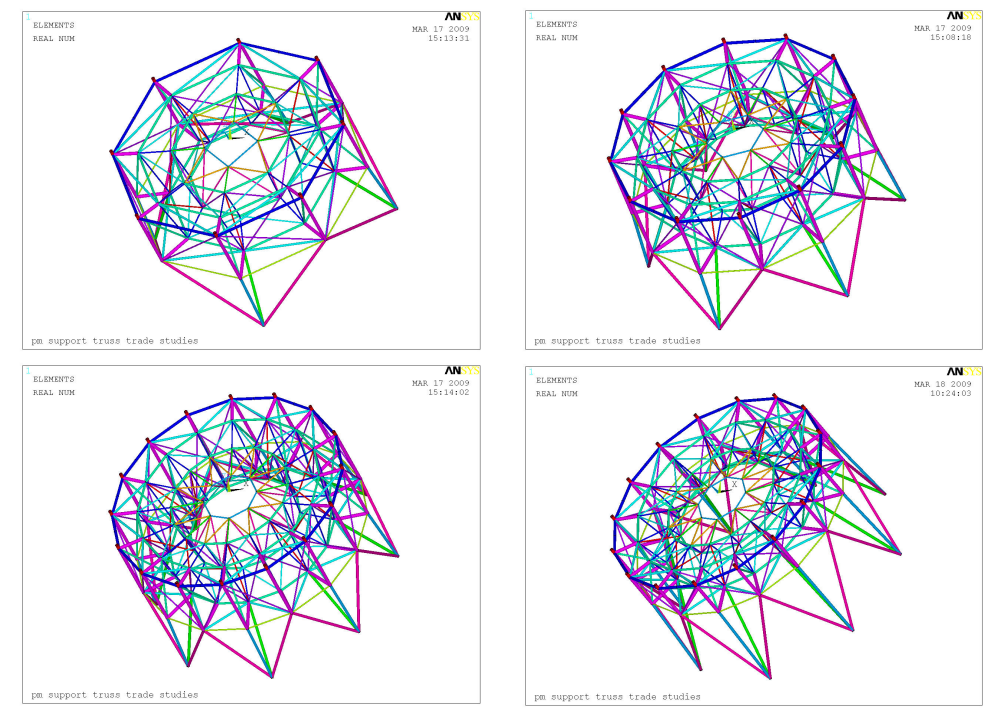

Fig. 5. This is a sample of the Base Truss trade studies conducted for the ATLAST $8 \mathrm{~m}$ Space Telescope. The main variable is the number of circumferential "cells" in the pattern. The depth of the truss sections is the next most important variable. The individual cross sectional properties (diameter and wall thickness) were the final variables, with similarly oriented tubes having the same properties. The PAF structure was designed integrally with this truss.

\subsection{Optical testing and relationship to support locations}

The gravity sag issue is mostly the cost of confidence. To put it another way, the more positions (and rotations) you take in a Schwesinger style mount, the more confidence you will have in the results. If the uniform spacing variant of Schwesinger is used, then all one has to do is build into the test support (tip-up frame etc), the ability of rotating relative to gravity. This may involve laying it on its' back and rotating, then tipping back up. Not a cheap solution, but within current engineering capabilities. To validate the model and mount, one varies the angle and magnitude of each (or selected) attachment points and compares wavefront errors to predictions. This alignment/force variation needs to be designed into the mount and the test program/schedules include this validation phase. The second orientation (horizontal mirror - vertical test tower), is also a cost versus accuracy/confidence issue. Enough support points, a lift/rotate system between mount and mirror to rotate mirror relative to mount (prior to bonding any pads to back) together with the proper design and calibration of the mount (proper sized hydraulic lines, diaphragm leakage control 
etc) and support point influence function testing (isolate, then increase force at selected points, and measure the wave front error induced and compare to model predictions. The cost is a function of the number of "correlation" tests and how good is good enough to believe the mount is functioning properly and that the results (with point-to point sag analyzed out) are believable.

With two separate tests and test mounts, and multiple ways to "validate" the mount error itself for both mounts, it should be possible to get community consensus on the results. Also, multiple null lenses and a point of curvature test to validate the null lenses are in order (just to avoid a Hubble style situation).

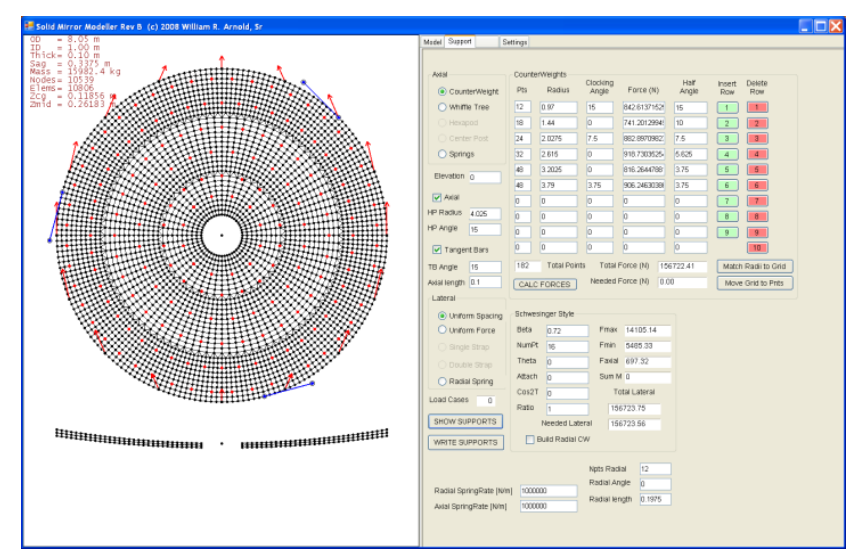

Fig. 6. Customized programs exist to design Thin Meniscus Mirror Support Systems. This particular program creates ANSYS input decks and permits many variations to be evaluated in a minimal amount of time. It is a third generation version of a system originally developed for L3 Comm - Brashear Div. of Pittsburgh, Pa. The USA based company which manufactured the Subaru primary mirror, one of the smoothest large mirrors ever made.

By designing access "channels" into the truss to permit a version of the Schwesinger mount to be applied to the full telescope assembly, then a full up system test is practical. It is not practical to include a hydraulic test mount in the launch support system (even if one could "drain" it prior to launch). The other option of a separate system behind the mirror and removal prior to launch is also not realistic. But a Schwesinger mount only requires cables for tensile (upper half) and rods for lower half. The length is not important, only the position and direction vector. A system can be designed to "miss" all the structural tubes of the metering truss and the radial constraint system can be designed around any access required. The same system can be tested at the component level (even if a greater number of points system is used to qualify the mirror, a subset can be used for full system testing ... as all the correlation data will exist).

The backing stiffness of the Base Truss can be verified with the aluminum surrogate mirror and concrete or steel dead weights to simulate satellite load at one gravity. A second point at say two gravities could be done easily with added weights at satellite points and sandbags (26 tons worth, spread over surrogate). A hydraulic test rig could also be used. Of all the telescope structure, this truss and its deflection are the most critical to the safety of the mirror. With launch supports in place, the displacement characteristics of truss are imprinted onto mirror as global displacement. How smooth this bending is and the magnitude must agree with analysis. The earlier a full mock up prototype can be made the better. A subscale 1/2 might work, with some typical full size truss segments tested as well.

\section{SECONDARY MIRROR SPIDER DESIGN INFLUENCE}

While the primary mirror support drove the basic truss design, mainly the number of circumferential cells in the truss, the support of the secondary mirror is the next most critical design driver. The spacing between the primary and secondary mirrors in a telescope controls the optical quality. The structure which maintains this spacing is known as the metering truss, and has various possible forms. The structure which spans the distance from the outer diameter of the clear aperture to the center support structure of the secondary is known as the spiders or spider arms. The number, width 
and spacing of the arms influence the optical performance through diffraction and obscuration. The ARES V shroud design places geometric constraints on the metering structure. Beyond the optical constraints on the structure, there are geometric and frequency requirements. While the primary mirror was the hardest design problem, the next most difficult problem was the metering structure between the primary and secondary mirrors. Optically, this is the most critical positional tolerance the telescope structure has to maintain. The interior volume and dimensions of the ARES V shroud (dynamic envelop) defines the trade space. The variables are how much of the cylindrical length available is allocated to Base Truss depth, Metering Truss cylindrical section and spider. It is possible to suspend the secondary on as few as three combination spider-metering arms as the JWST design has done.

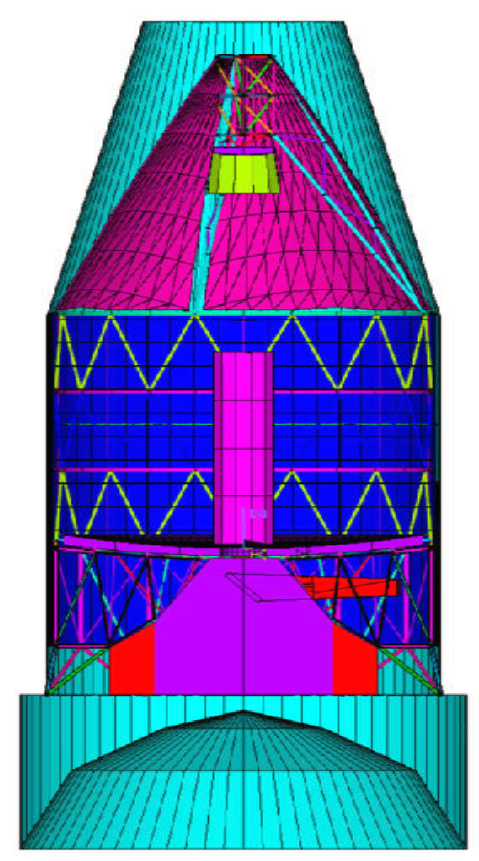

BICONIC SHROUD

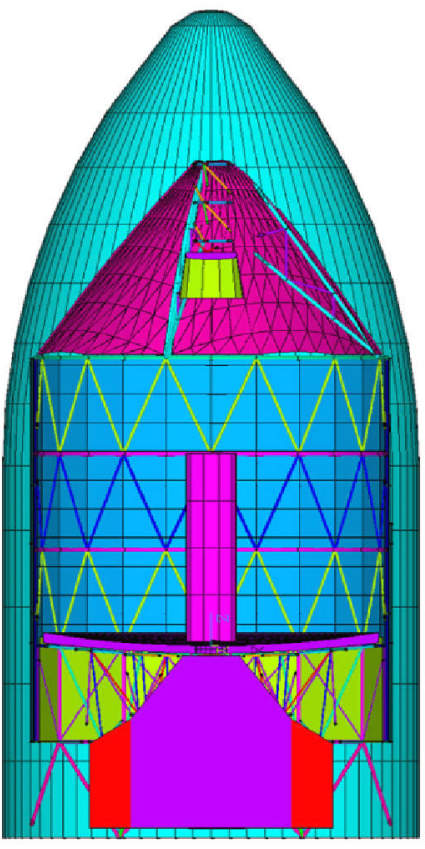

OGIVE SHROUD

Fig. 7. ATLAST must fit within the dynamic envelop of the ARES V shroud. The structural design must continually allocate the available volume between the satellite, instruments and optical assembly needs. (VIEWS NOT TO SAME SCALE)

The problem comes in the lateral vibration modes of these arms. The more of the primary to secondary spacing done only by the spider arms, the lower the natural frequencies possible. To raise the natural frequency, you must widen the arm, obscuring more light. The minimum spider arm length is half the distance of primary mirror diameter minus secondary mirror diameter that sets the minimal width possible. The Hubble metering truss is essentially a truss implementation of a cylinder, the rings defining the cylinder bore and the cross bracing elements providing the axial and torsional stiffness. The oil can modes of the cylindrical metering truss are a function of the ring cross sectional properties, the number of segments and the angles of the axial cross-members. The ANSYS models of the metering structure have all these variables in their parametric capabilities. Secondary consideration of the metering truss ring "thickness" is how much of the radial difference between clear aperture of the primary mirror and the inside of the dynamic envelop is allocated to the structure and how much is available for a deployable sun shield. The shroud interior does not allow a full length Hubble style truss, and the natural frequency minimums do not allow a JWST style spider, so a compromise is necessary.

How "fast" the optical system is effects the quality of telescope and the cost. While space optics have been built with very fast (i.e. spacing of the primary to secondary relative to the diameter of the primary), the faster the mirror, the rougher is gets due manufacturing realities. We have elected for this study to restrict ourselves to "slower" mirror 
prescriptions, where the existing manufacturing techniques can produce UV quality results. Hence we have concentrated on a hybrid design as should in Figure 7.

Two separate shrouds were considered in the trade studies, the baseline Bi-conic and the Ogive. It was possible to keep the same optical prescription in both cases, but the Bi-conic variation has lower minimum lateral frequencies due to longer spiders and a slightly reduced instrument volume. 
The following series of figures shows representative variations of the metering truss and spider design variables. Beyond the three versus four spider arms of the baseline study, a series of side studies were conducted to look at TPS (Terrestrial Planet Finder) applications. This involved curved spiders, movable spiders to create large diffraction free portions of the clear aperture. By the end, every variation from single to four arms was evaluated, both in launch configuration and on orbit jitter performance. While it might be possible to get one of these schemes to work, they
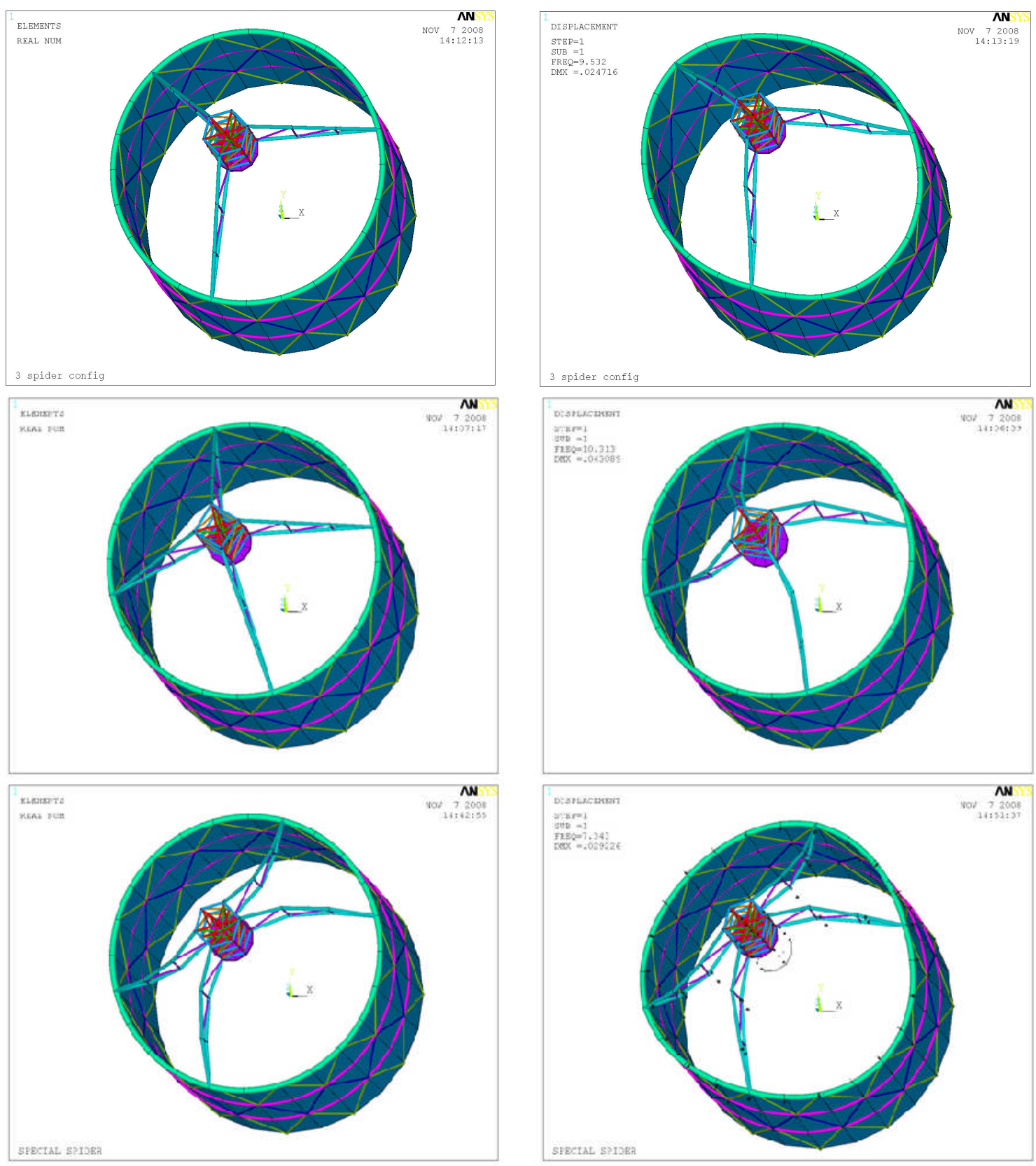

would severely compromise the performance of the structural system and require complex mechanisms to get through launch. 
Fig. 8. These are the basic spider configurations. The three arm version has the lowest obscuration, but requires a more massive head ring to control the "oil can" modes of the cylindrical section. The four arm version has better dynamics and door configuration, but has higher light blockage.
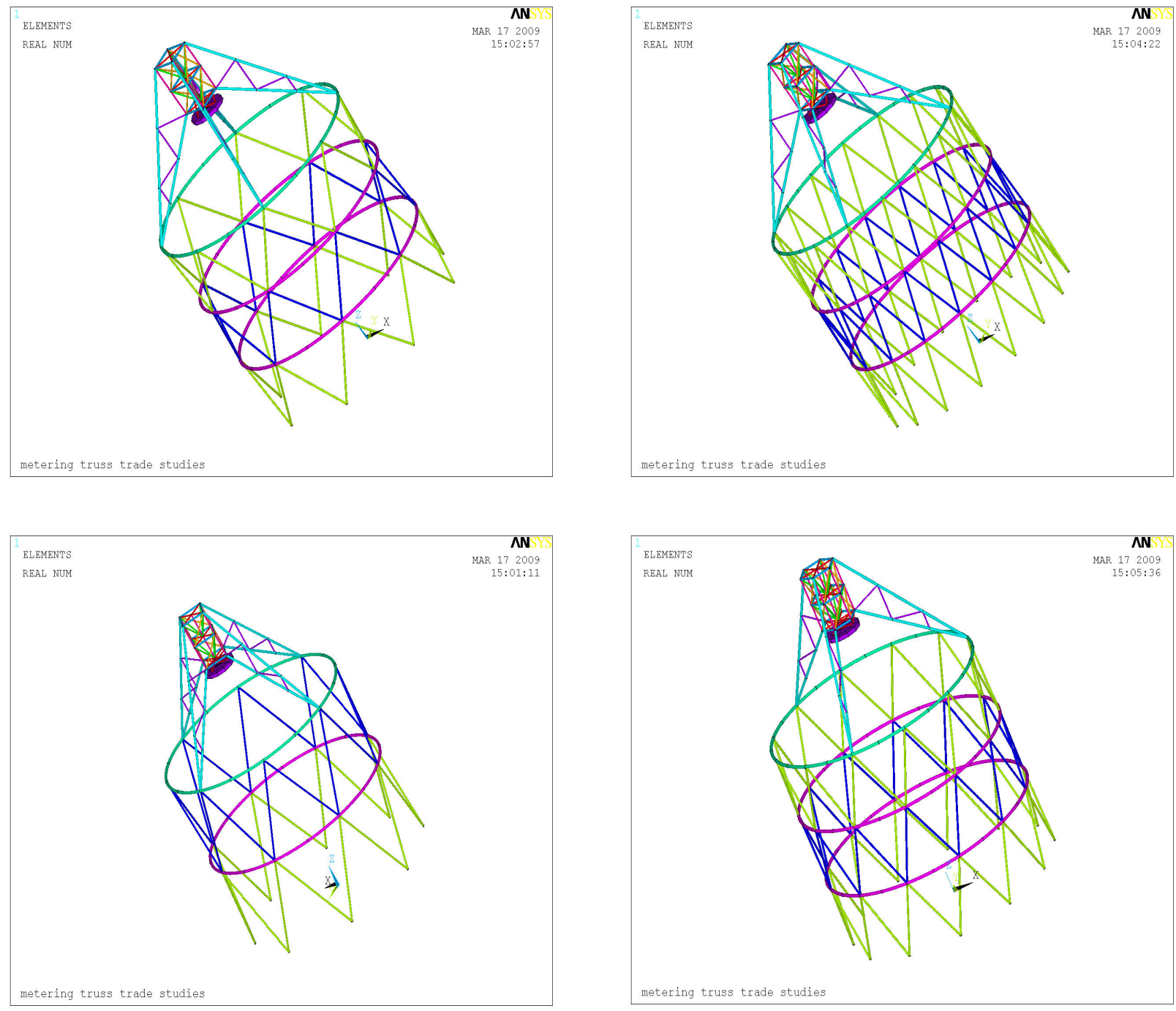

Fig. 9. Trade studies were conducted on the metering truss and spider configuration. These are some of the typical variations looked at. In the end, the primary mirror Base Truss geometry narrowed the possible candidates as the number of truss intersections at the common plane between Base Truss and Metering Truss has to match. 


\section{CONCLUSIONS AND FURTHER WORK}

It is feasible to launch an 8-meter class monolithic meniscus mirror on the ARES V launch vehicle.

It is feasible to test and qualify such a telescope system

Such a telescope will open new vista for astronomy in the areas of .....

Key areas for further research and development towards this mission are:

(1) Refine the concepts for launch support systems (radial and axial)

a. Detailed design work

b. Prototype testing of likely candidates

(2) Establish community consensus on approaches to vibro-acoustic testing of the system

a. Use of aluminum surrogate primary mirror

b. Correlation of subscale and simulations

c. Contamination and FOD control if actual mirror must be used.

(3) Development of coating uniformity techniques for 8 meter class optics.

(4) Further mission planning and "guidelines" for design of robotic serving friendly modules.

\section{REFERENCES}

[1] Stahl, H. Philip and the ATLAST Study Team, "Design of an 8-meter Monolithic UVOIR Space Telescope", AAS Winter Meeting, January 2009.

[2] Stahl, H. Philip, Phil Sumrall, and Randall Hopkins, "Ares V launch vehicle: an enabling capability for future space science missions”, Acta Astronautica Elsevier Ltd., 2009, doi:10.1016/j.actaastro.2008.12.017.

[3] Stahl, H. Philip and Marc Postman, "8 meter Monolithic UV/Optical Space Telescope", 59th International Astronautical Congress, Glasgow, Scotland, Sept 2008.

[4] Stahl, H. Philip, "Design study of an 8 meter monolithic mirror UV/optical space telescope", SPIE Astronomy Conference, Marseille, France, June 2008.

[5] Postman, Marc, et. al., "A Large Monolithic-Aperture Optical/UV Serviceable Space Telescope Deployed to L2 by an Ares-V Cargo Launch Vehicle", Science Associated with Lunar Exploration Architecture, Tempe, AZ Feb. 28, 2007.

[6] Nelson, Jerry E., Jacob Lubliner and Terry S. Mast, "Telescope mirror supports: plate deflections on point supports", SPIE Vol. 332 Advanced Technology Optical Telescopes (1982), pp 212-228.

[7] Schwesinger, G., "Lateral support of very large telescope mirrors by edge forces only", Journal of Modern Optics, Vol. 38, No. 8, pp. 1507-1516, 1991.

[8] Gerhard Schwesinger, "Non-distorting lateral edge support of large telescope mirrors," Appl. Opt. 33, 1198-1202 (1994)

[9] ATLAST Astrophysics Mission Concept Study Final Report

${ }^{[10]}$ http://www.stsci.edu/institute/atlast/index html ATLASTMissionConceptStudy Page 


\section{STRUGTURAL DESIGN CONSIDERATION.} FOR AN 8M SPACEITELESCOPE

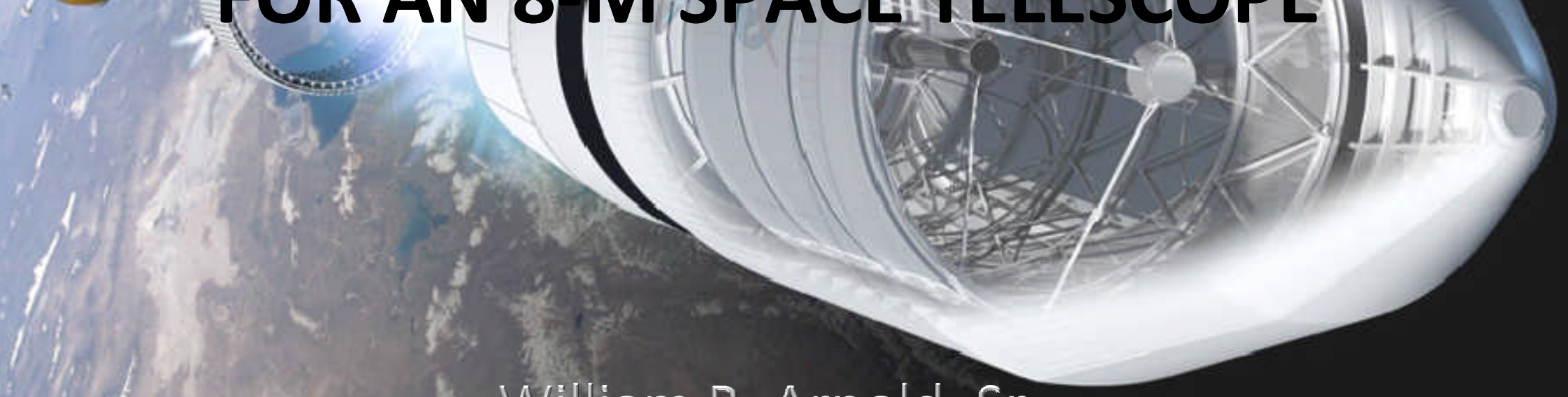

William R. Arnold, Sr.

H. Philip Stahl 


\section{WHAT ARE WE GOING TO TALK ABOUT}

- Advantages of using big meniscus mirrors in a space telescope

- What are the major elements in a space observatory

- What are the design drivers (particularly for 8-m thin meniscus mirror systems)

- How these requirements drive the focus on primary mirror suspension design

- The available "design space" or choices to be made.

- A look into the current baseline for an 8-m system

- Where do we go from here? 


\section{INTRODUCTION}

- POSSIBILITIES OPENED WITH ARES V VEHICLE

- PAYLOAD CAPACITY

- SHROUD VOLUME

- TRADITIONAL SPACE MIRROR COMPROMISES

- MACHINED AND/OR CAST LIGHTWEIGHTS

- EGGCRATES (FRIT OR FUSION BONDED)

- SEGMENTED MIRRORS

- EXOTIC MATERIALS (HIGHER CTE)

- FABRICATION ADVANTAGES OF THIN MENISCUS

- SMOOTHNESS A FUNCTION OF QUALITY OF MOUNT

- TECHNOLOGY MATURE FOR LARGE MIRRORS

- MECHANICAL ADVANTAGES OF THIN MENISCUS

- UNIFORM GLOBAL BENDING CHARACTERISTICS

- PEAK STRESS AT SURFACE WHERE PROCESS CAN IMPROVE STRENGTH 


\section{BASIC ELEMENTS OF A SPACE TELESCOPE}

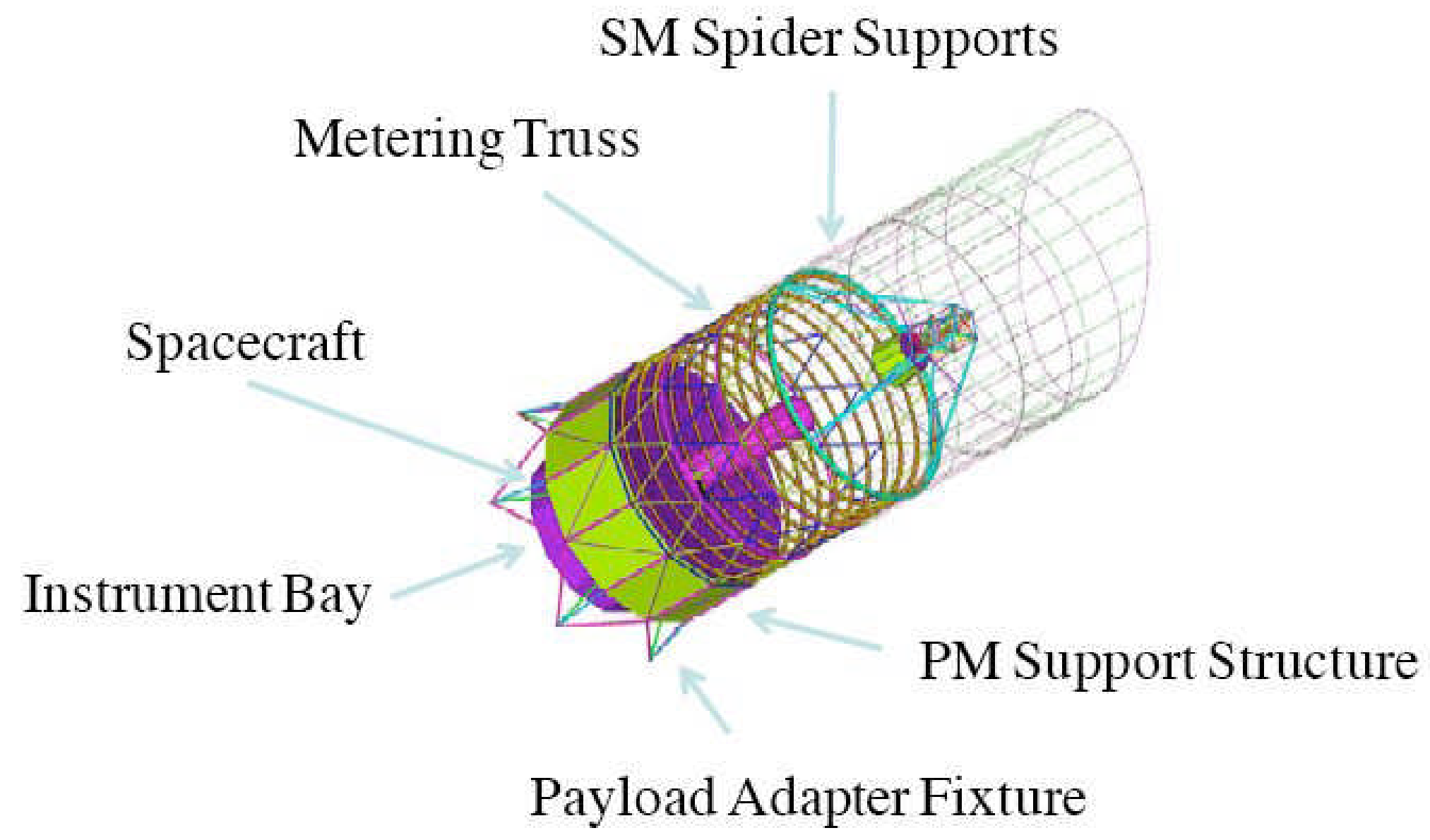




\section{ATLAST OPTICAL LAYOUT}
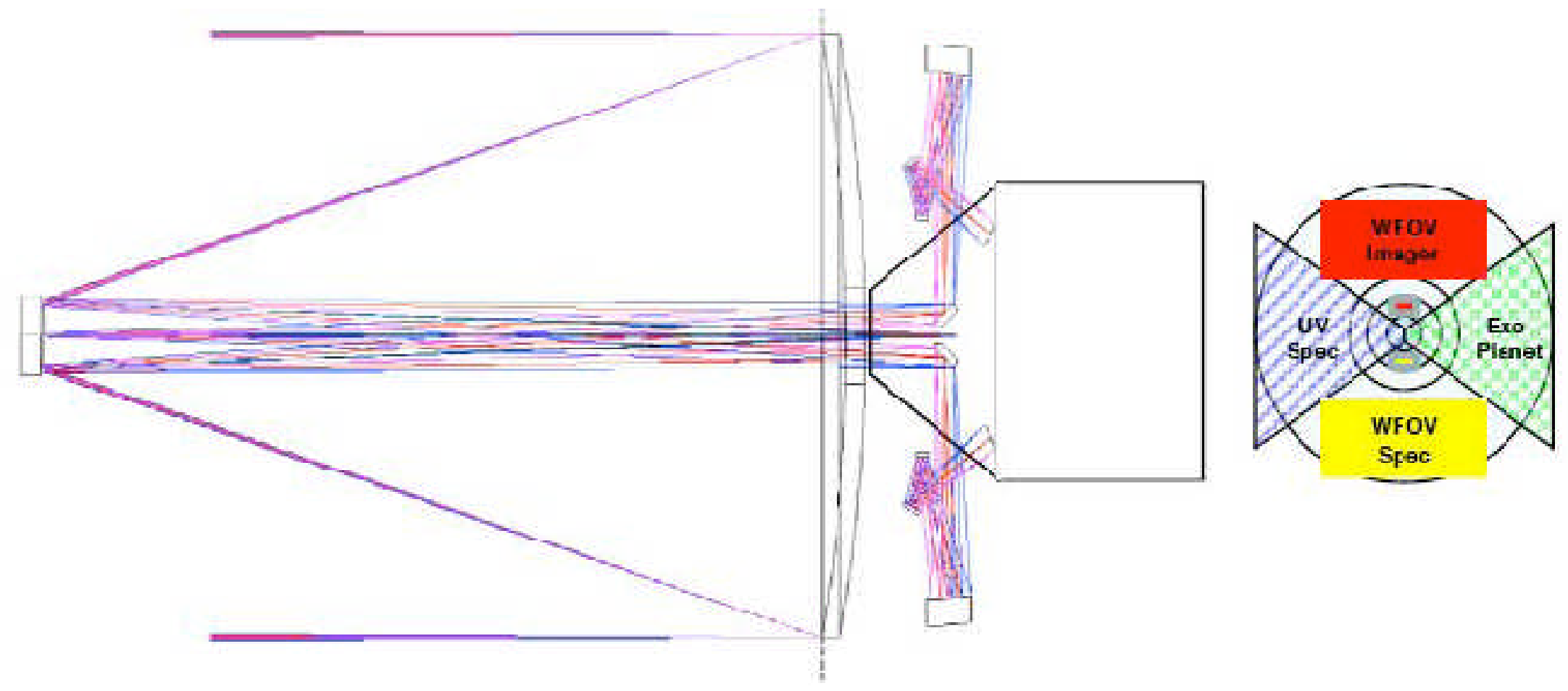

The heart of any telescope is the optical layout. The ATLAST design allows for multiple uses and future expansion of capabilities. 


\section{MODULAR SPACECRAFT DESIGN}

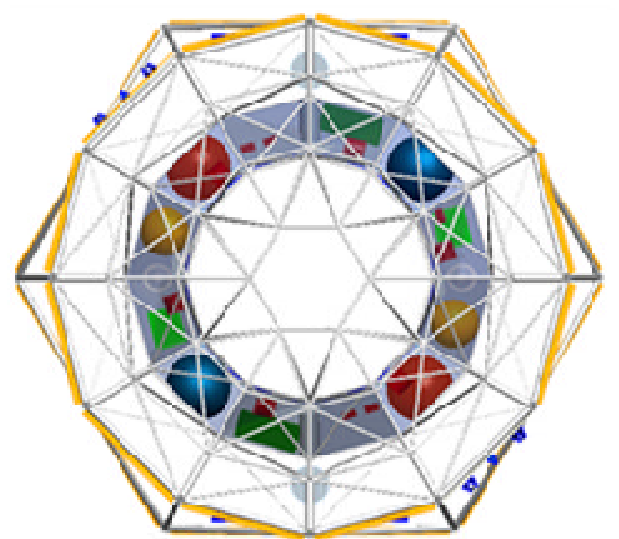

Top View

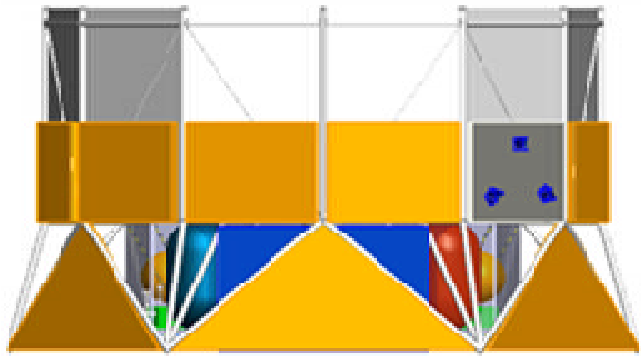

Frand Wian

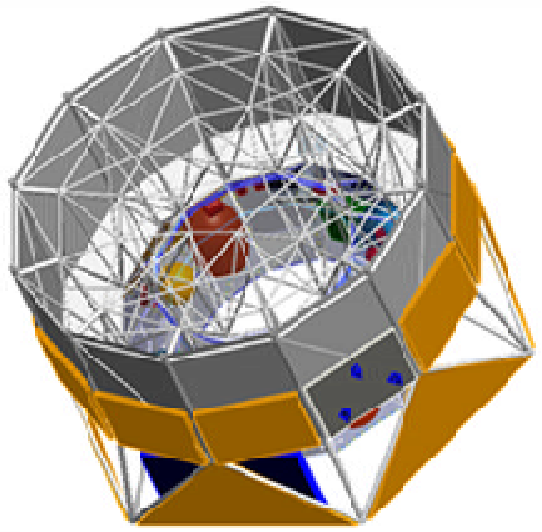

Is0 Viaw

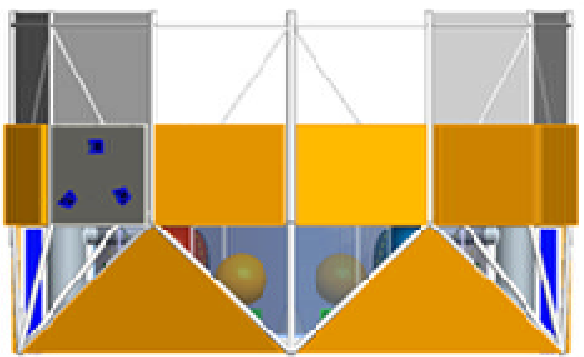

sille viaw

From the beginning, the servicing and capability updates are designed into the system. 


\section{DESIGN DRIVERS \& IMPORTANT ISSUES}

- BASIC DESIGN DRIVERS

- PRIMARY MIRROR SUPPORT SYSTEMS REQUIREMENTS

- TELESCOPE STRUCTURE REQUIREMENTS

- SATELLITE AND INSTRUMENT REQUIREMENTS

- TESTING AND VERIFICATION

- UNRESOLVED ISSUES AT THIS POINT 


\section{BASIC DESIGN DRIVERS}

- Survive the launch environment

- Not interfere with Launch Vehicle control systems (Minimum frequency requirements)

- Be within the lift capacity of the vehicle and fit within the shroud dynamic envelop

- Get into operational configuration (deployment, release mechanisms, etc.)

- Function in operational environment (thermal, jitter and optical performance)

- Serviceable and/or sustainable over planned life (redundancy, replacement and replenishment) 


\section{PRIMARY MIRROR SUSPENSION REQUIREMENTS}

- The support system must retain the mirror throughout the trajectory, while subject to accelerations, vibrations and acoustical loads.

The allowable stress in the mirror is a function of surface finish on back of the mirror.

- The support system must couple or decouple the primary mirror as a mass-spring system from the frequency range of the launch vehicle major structural and control frequencies.

Usually stated as $\mathbf{2 0}$ hertz for axial and 7-8 hertz lateral.

- The support system must release the mirror to a kinematic state after achieving orbit; this kinematic system must control the position of the mirror during insertion and operational maneuvering

If any of the launch system stays in contact with the primary, it will degrade the optical performance by causing bending in the mirror. 


\section{TESTING AND VERIFICATION}

- Technology to make big mirrors is well established ... but!

- No one has launched any mirror this massive

- Want to be able to test optically at component and system level

- Want to test mechanically, but cost, schedule and complexity of working with a finished optic this size says another approach is called for.

- Every step in fabrication, test and assembly involves massive tooling, so you have to plan ahead.
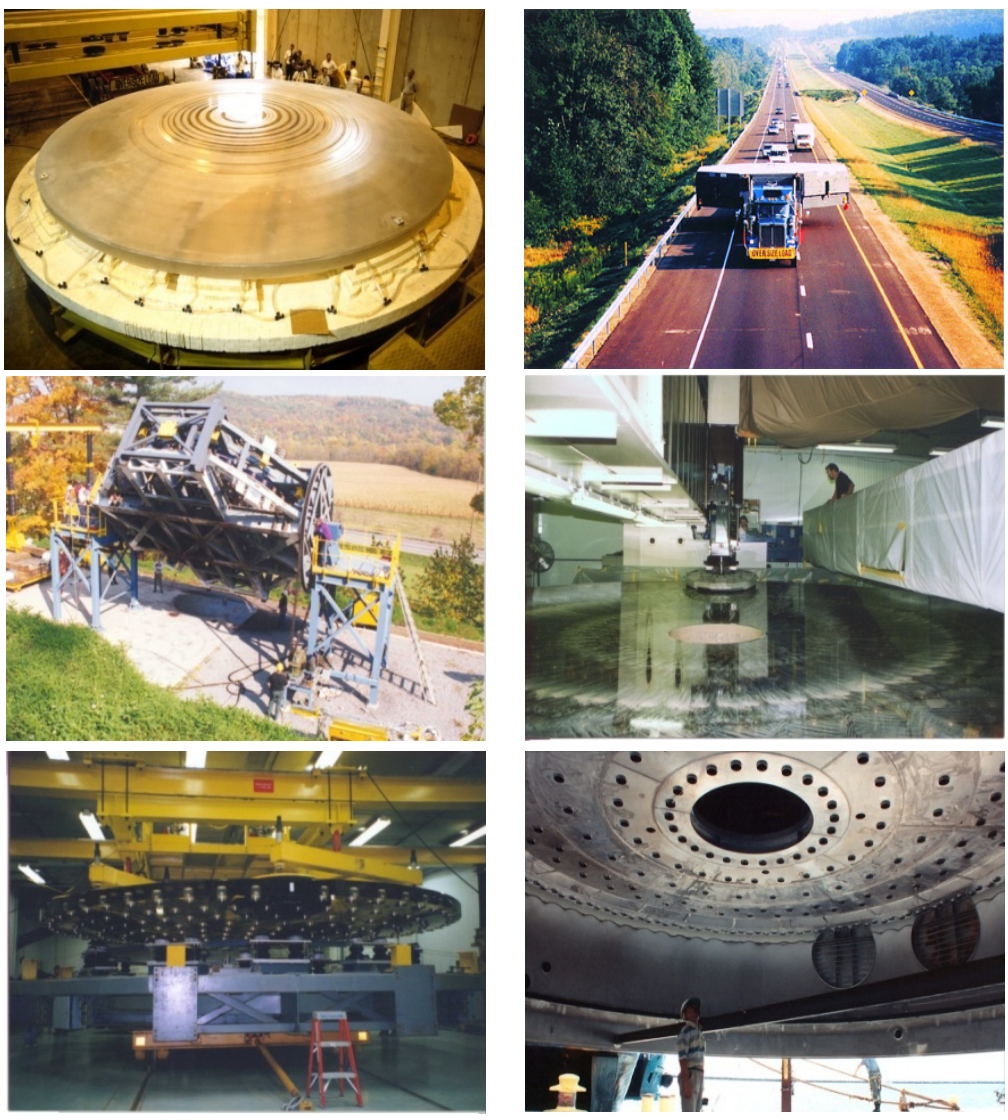

Subaru 8.3m Primary Mirror under Construction (Photos courtesy of L3-Comm Brashear Division) 


\section{SPECIALIZED TOOLS TO DESIGN SUPPORTS}

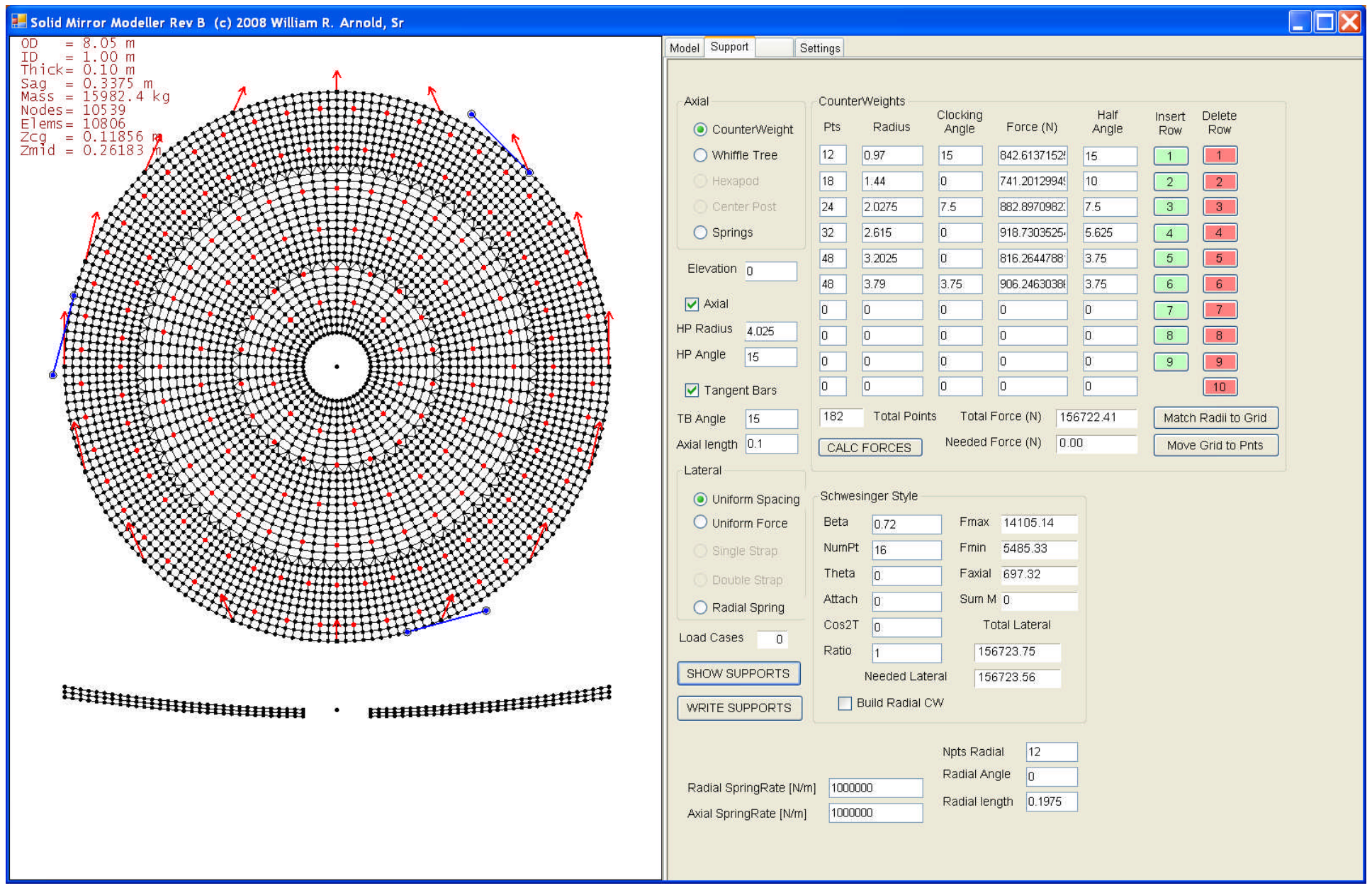




\section{PRIMARY MIRROR SUSPENSION SYSTEMS}

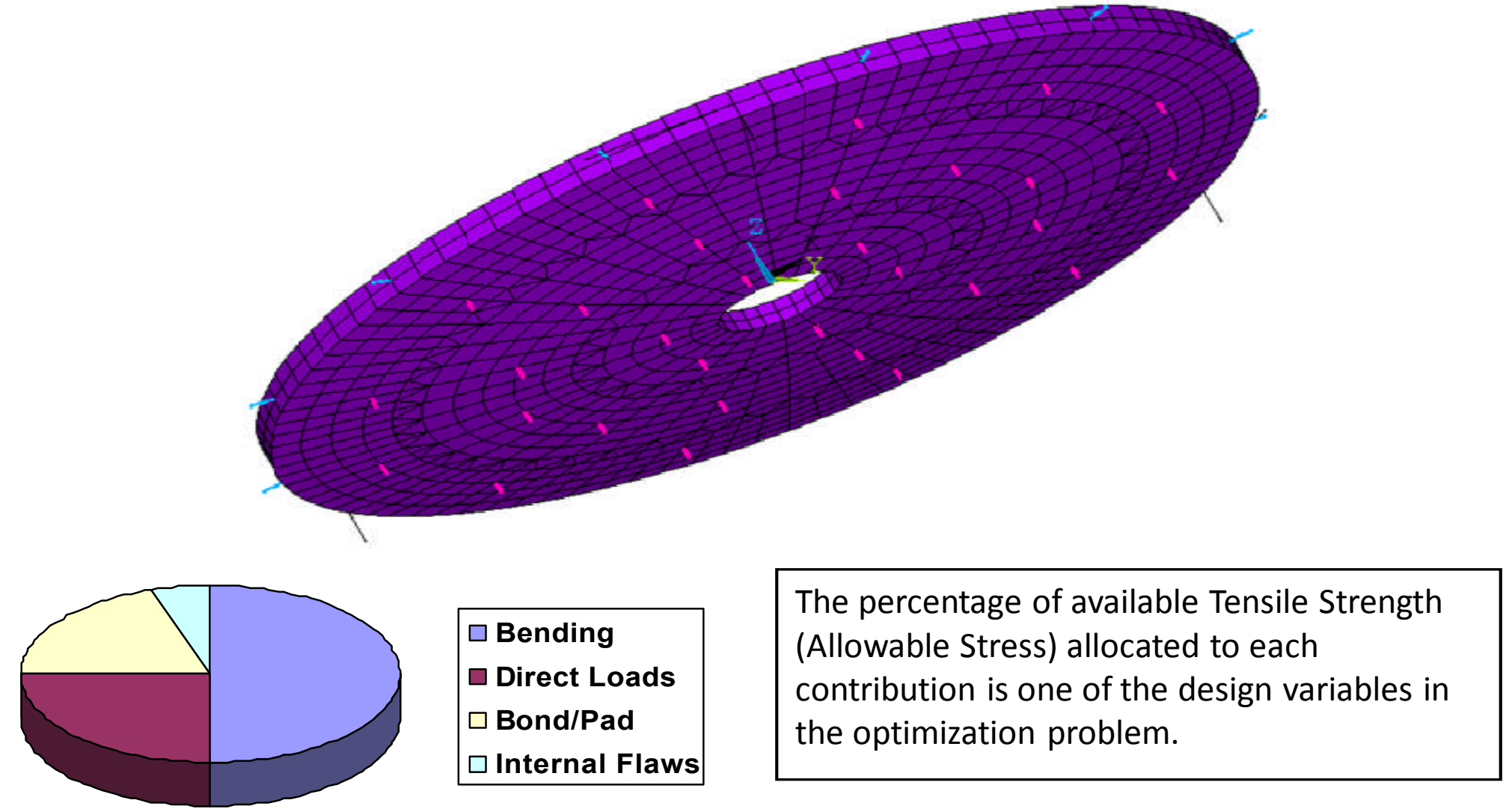

Practical versus desirable suspension systems, what works on earth will not work for the launch environment or on orbit. We need to understand this point. 


\section{BASE TRUSS VARIATIONS}
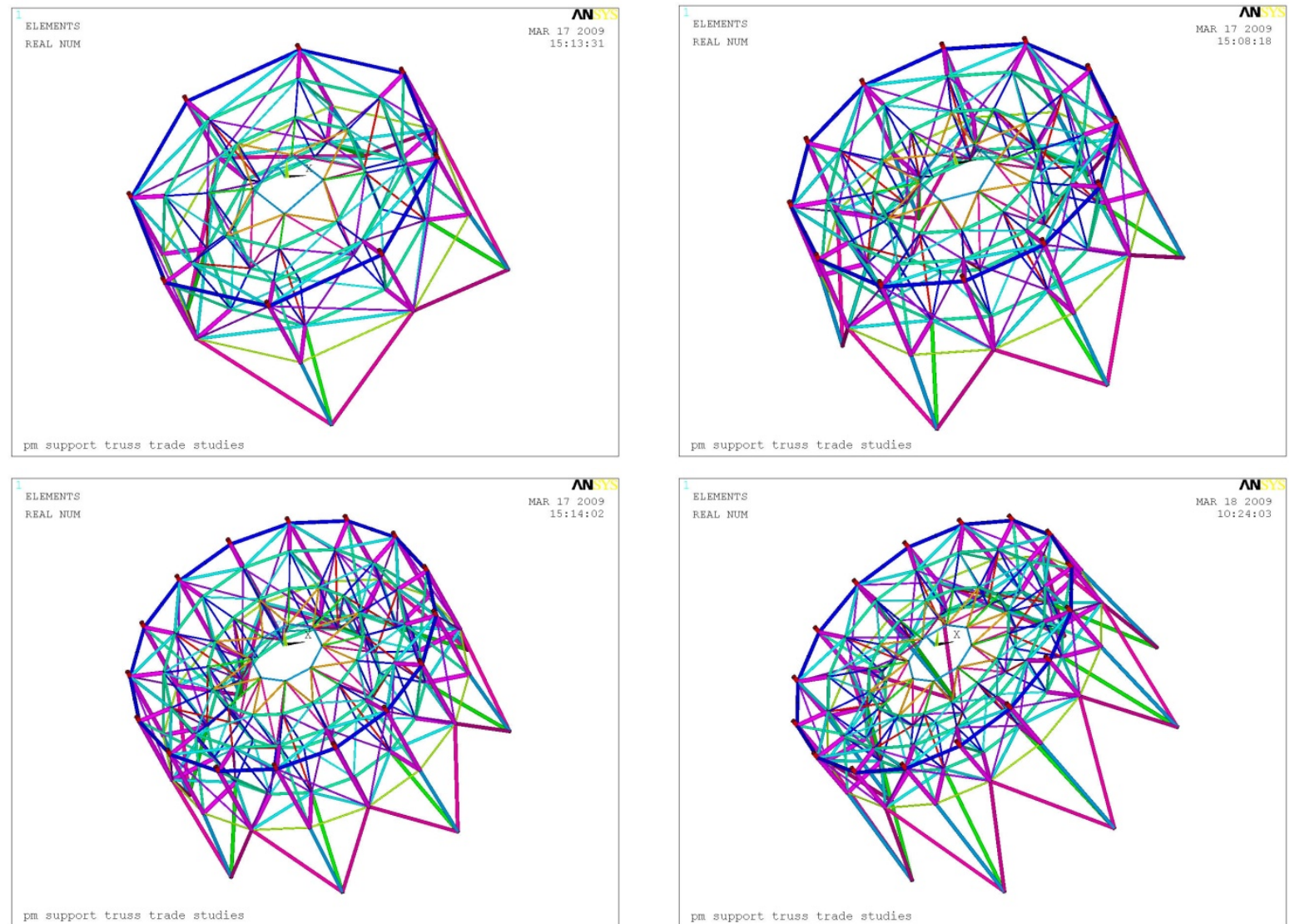

The mirror support locations have to line up with truss intersections for best stiffness characteristics, plus truss must carry satellite any not excessively bend mirror during launch. 


\section{SHROUD BASED TRADE STUDIES}

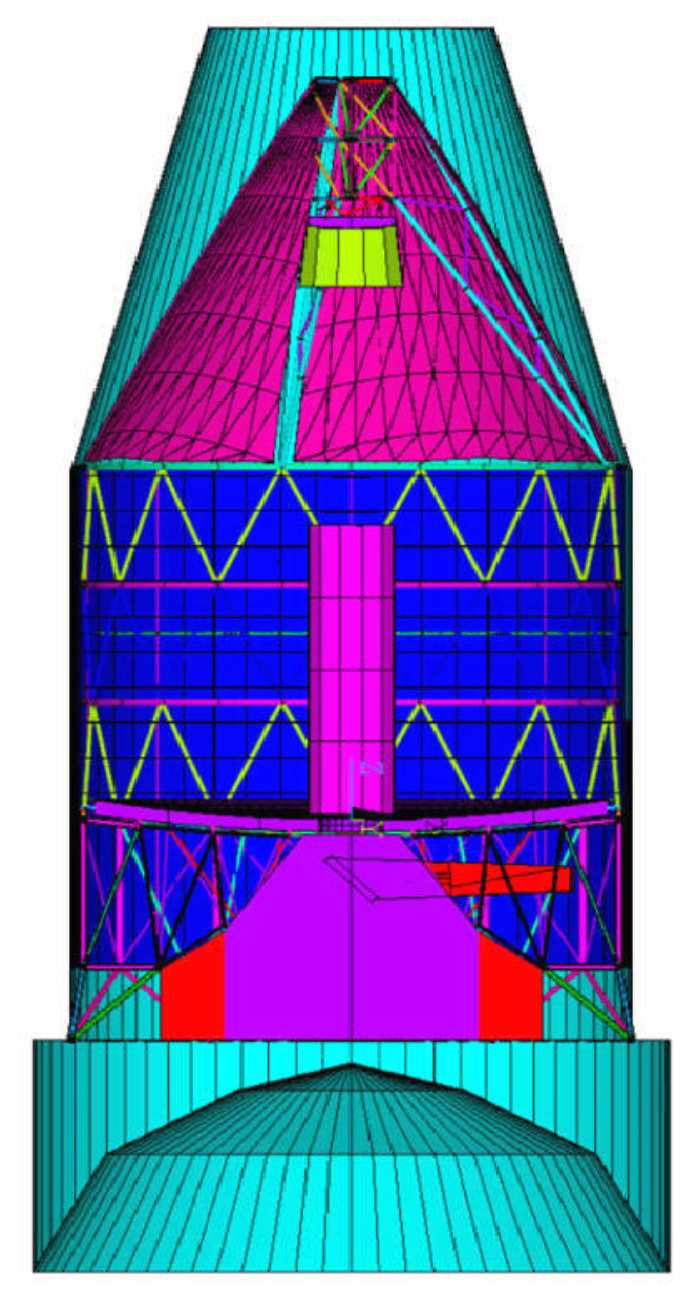

BICONIC SHROUD

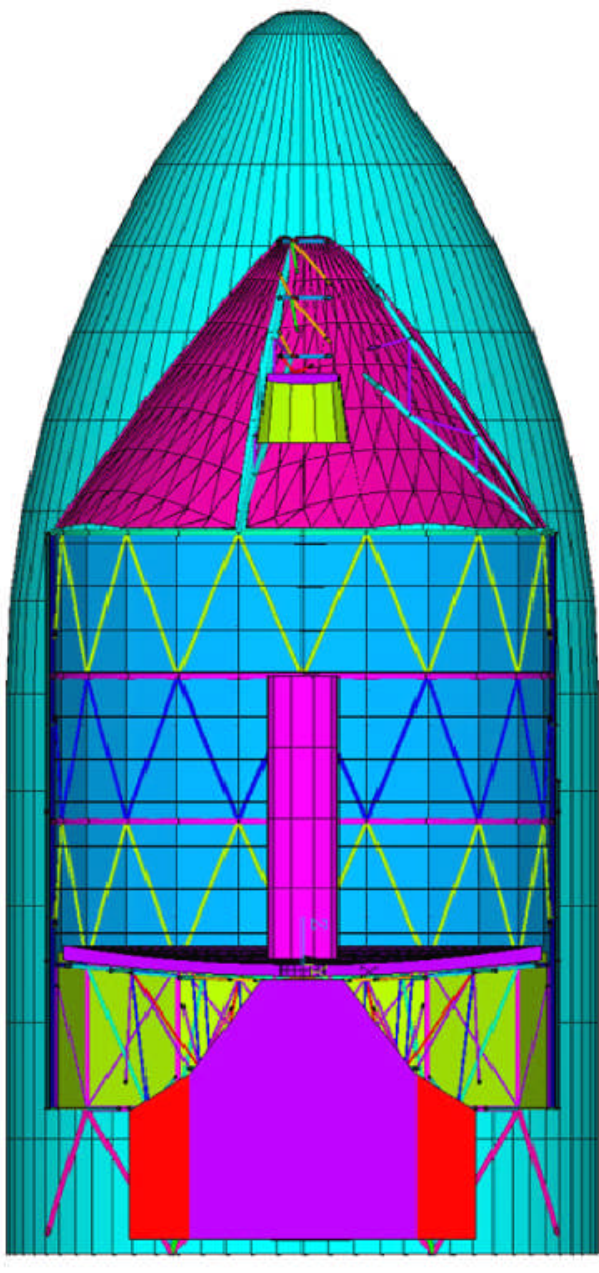

OGIVE SHROUD 


\section{METERING TRUSS - SPIDER STUDIES}
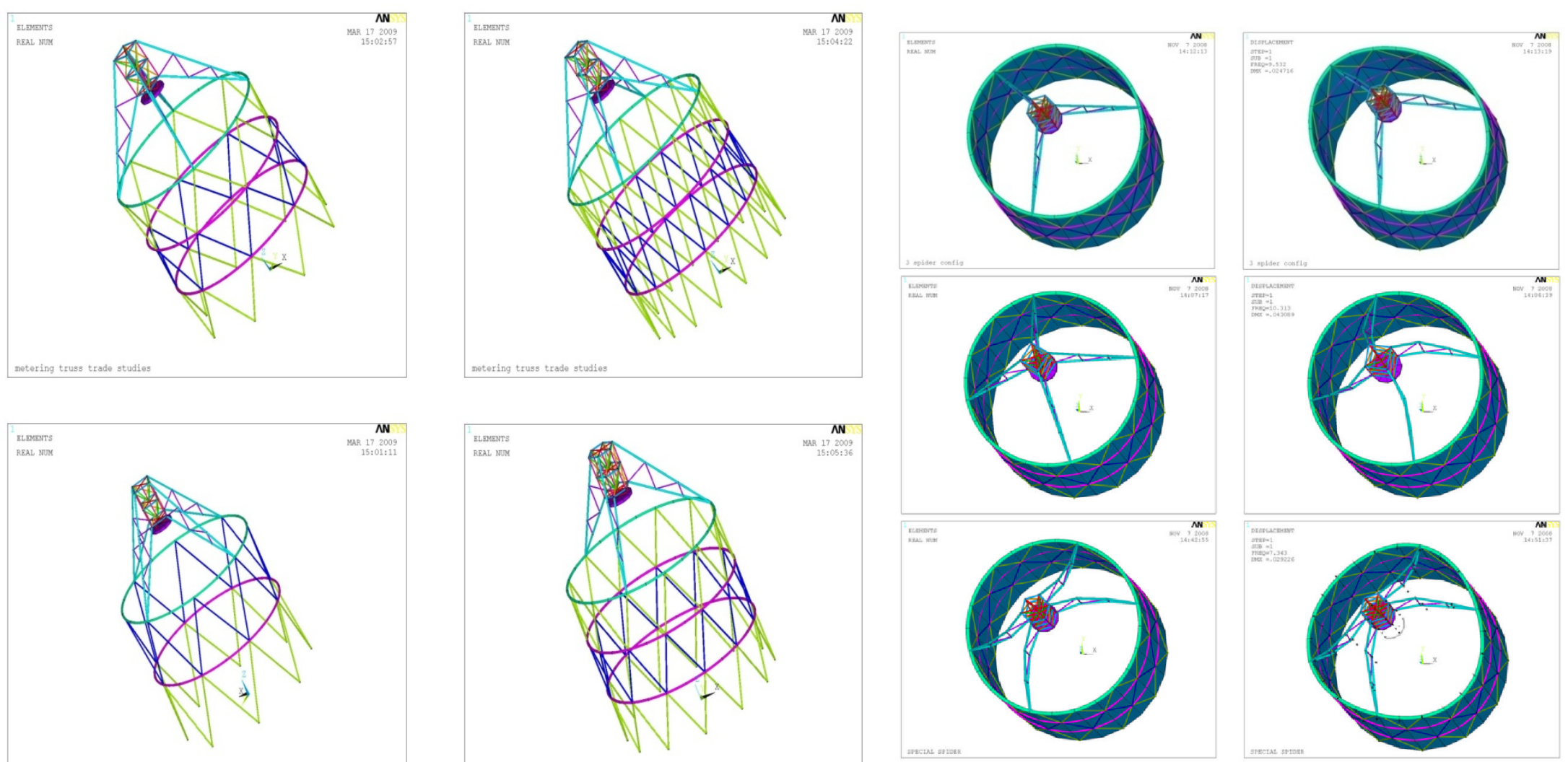

The metering truss attachment points have to match the Base Truss geometry. The balance of spider and truss frequencies drives the design. 


\section{CONCLUSIONS}

It is feasible to launch an 8-meter class monolithic meniscus mirror on the ARES V launch vehicle.

$\square$ It is feasible to test and qualify such a telescope system

$\square$ Such a telescope will open new vista for astronomy in the areas of ..... 


\section{KEY AREAS FOR FURTHER RESEARCH \& DEVELOPMENT}

$\square$ Refine the concepts for launch support systems (radial and axial)

- Detailed design work

- Prototype testing of likely candidates

$\square$ Establish community consensus on approaches to vibro-acoustic testing of the system

- Use of aluminum surrogate primary mirror

- Correlation of subscale and simulations

- Contamination and FOD control if actual mirror must be used.

$\square$ Development of coating uniformity techniques for 8 meter class optics.

$\square$ Further mission planning and "guidelines" for design of robotic serving friendly modules. 